\title{
Radio emission from AGN detected by the VLA FIRST survey ${ }^{\star}$
}

\author{
Y. Wadadekar ${ }^{\star \star}$ \\ Institut d'Astrophysique de Paris, 98bis boulevard Arago, 75014 Paris, France \\ Space Telescope Science Institute, 3700 San Martin Drive, Baltimore, MD 21218, USA
}

Received 29 August 2003 / Accepted 19 November 2003

\begin{abstract}
Using the most recent (April 2003) version of the VLA FIRST survey radio catalog, we have searched for radio emission from > 2800 AGN taken from the most recent (2001) version of the Veron-Cetty and Veron AGN catalog. These AGN lie in the $\sim 9033$ square degrees of sky already covered by the VLA FIRST survey. Our work has resulted in positive detection of radio emission from 775 AGN of which 214 are new detections at radio wavelengths.
\end{abstract}

Key words. galaxies: active - methods: statistical - catalogs - surveys

\section{Introduction}

Systematic radio observations of samples of active galaxies (AGN) have a long history (throughout this paper we use the term AGN to designate all active galactic nuclei, except quasars). De Bruyn \& Wilson $(1976,1978)$ surveyed a sample of 43 Markarian Seyferts and found that Seyfert 2 galaxies have on the average a higher radio luminosity than Seyfert 1 galaxies. Ulvestad \& Wilson (1989) used high resolution data from the VLA on a radio flux limited sample of 79 Markarian Seyferts and a distance limited sample of 25 Seyfert galaxies. They also found that Seyfert 2 galaxies are systematically more luminous than Seyfert 1 galaxies and that a weak correlation exists between sizes and powers of nuclear radio sources in Seyferts. On average, Seyfert 2 radio sources were found to be larger than those of type 1 Seyferts. However, in a subsequent study, Ulvestad \& Wilson (1989) found that their sample of Seyfert 2's could have been biased in radio luminosity. Their subsequent analysis of a distance limited sample of 27 Seyfert galaxies that was free of this bias showed that the earlier conclusions were not statistically significant. This new result supported the finding of Edelson (1987) that there was no significant difference between the radio luminosities of the Seyfert 2 and Seyfert 1 galaxies in his magnitude limited sample of 42 Seyferts. More recently, Ulvestad \& Ho (2001) found that Seyfert 1 galaxies host somewhat stronger radio sources than Seyfert 2 galaxies at 6 and $20 \mathrm{~cm}$, particularly among the galaxies with the weakest nuclear activity. They used a sample of 45 Seyfert galaxies from the Palomar spectroscopic survey of nearby galaxies.

^ Tables 3 and 4 are only available in electronic form at the CDS via anonymous ftp to cdsarc.u-strasbg.fr $(130.79 .128 .5)$ or via http://cdsweb.u-strasbg.fr/cgi-bin/qcat?J/A+A/416/35

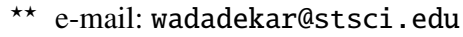

The aim of many of the above studies was to use statistical properties of radio sources in these galaxies to draw conclusions about their physical character and to test the unification scheme. According to the unification scheme for AGN, at a given intrinsic luminosity, all other properties of AGN from spectroscopic classification to VLBI component speeds can be ascribed to orientation effects.

A straightforward test of the unification hypothesis is to see if a property that is not orientation dependent has similar values and distribution for different classes of AGN. Assuming that the standard unification scheme for AGN is valid, radio and FIR luminosities of AGN are expected to be orientation independent, except over a small angle where Doppler boosting of radio jets occurs. This is because neutral gas and dust surrounding the radio source itself cannot obscure it, and even ionized gas (found in nuclear starbursts) is transparent at radio frequencies $v>3 \mathrm{GHz}$, for AGN with low to moderate emission measure. In fact, the most compact nuclear starbursts are transparent only at $\mathrm{GHz}$ radio and far infrared (FIR) wavelengths. At other wavelengths, absorption of the radiation by the intervening matter surrounding the nucleus is significant and it is very difficult to measure the intrinsic luminosity. In principle, the unification scheme can be tested by radio observations of different kinds of AGN.

In the standard unification scheme for Seyferts, it is expected that Seyfert 1 and Seyfert 2 galaxies should have similar total radio power (since the radio emission is largely unobscured at centimeter wavelengths). Also, if the predominant radio emission is from jets, radio jets from Seyfert 1 would be foreshortened as they are viewed closer to end-on. Statistically, this implies that Seyfert 1 radio source sizes should be smaller than Seyfert 2 sizes. Schmitt et al. (2001) have found this effect in a sample of Seyfert galaxies selected at $60 \mu \mathrm{m}$. 
Table 1. Summary of radio detections.

\begin{tabular}{lc}
\hline \hline Number of VV01 AGN in FIRST area & $\sim 2840$ \\
Number of AGN with radio detections & 775 \\
Number of non-detections & $\sim 2065$ \\
Percentage of AGN with radio emission & \\
detected by FIRST & $27 \%$ \\
\hline
\end{tabular}

All the previous studies of radio emission in AGN have suffered due to their small sample sizes (typically 50 galaxies). Some of them have also been affected by subtle selection biases that were only discovered many years later. In this work, we approach the problem from a different perspective. We use data from the recently completed VLA FIRST survey to look for radio emission from all known AGN, lying in the VLAFIRST survey area. Our sample size is an order of magnitude larger than typical sample sizes in previous studies. However, it is by no means a well defined complete sample, but is motley collection of active galaxies. It is a supersample of all previous samples, subject to all the selections biases and incompleteness of each of these. It is therefore, impossible to use our sample for construction of radio luminosity functions. The large sample size, however, may make it possible to recognize significant statistical trends in the data.

Our AGN sample is drawn from the most recent (10th) edition of the Veron-Cetty \& Veron (2001) catalog, which is a complete survey of the literature and lists all quasars and active nuclei known to the authors of the catalog prior to May 1, 2001. They define a quasar as a starlike object, or object with a starlike nucleus, brighter than absolute magnitude $M_{B}=-23$ (using $H_{0}=50 \mathrm{~km} \mathrm{~s}^{-1} \mathrm{Mpc}^{-1}, q_{0}=0$ ). Objects fainter than this limit appear in Table 3 of the catalog, which we have used in this work (hereafter VV01). Some of the objects listed in this table would move to Table 1 (which lists quasars) and vice versa for a different choice of cosmological parameters. Variability and the size of the diaphragm used for the apparent magnitude measurement may have a similar effect, as the contribution of the underlying host Galaxy may be significant for the faint quasars. Such a criterion has the additional shortcoming that at higher redshifts the AGN luminosity function is artificially truncated. However, these caveats in sample identification are offset by the large size of the sample - 5751 active galaxies are listed in Table 3 of the catalog, of which about 2840 lie in the area covered by the FIRST survey.

Deep, high resolution, large area radio surveys provide the most efficient means to detect radio emission from known AGN. The recently completed VLA Faint Images of the Radio Sky at Twenty centimeters (FIRST) survey (Becker et al. 1995; for more upto date information see the FIRST survey homepage at http://sundog.stsci.edu/) combines a large sky coverage with a low flux limit of $1 \mathrm{mJy}$ at $20 \mathrm{~cm}$. This survey now covers $>9000$ square degrees mostly around the North Galactic Cap with a small equatorial strip, the same area of the sky now being surveyed by the Sloan Digital Sky Survey (SDSS, http://www.sdss.org/). To date, data for approximately 9033 square degrees of sky have been released. The FIRST survey is now substantially complete. A small amount of additional data to fill holes within the surveyed area may be acquired; but an increase in the area covered is unlikely.

The FIRST survey has better sensitivity and resolution than its sister survey the NRAO VLA Sky Survey (NVSS, Condon 1998), although it covers a smaller area. More importantly, the FIRST survey, which is being carried out with the VLA in its B-configuration, has a resolution of 5 arcsec combined with excellent astrometric accuracy of $\sim 1^{\prime \prime}$ (90\% error circle) and a 5 sigma sensitivity of $\sim 1 \mathrm{mJy}$. This compares favorably with the D-array NVSS, which has a beam size of 45 arcsec and a 5 sigma sensitivity of $\sim 2.4 \mathrm{mJy}$. FIRST has a smaller beam size, a better resolution and more accurate astrometry than NVSS. It is therefore well suited for studies of radio emission from compact sources such as quasars and AGN. A study of radio emission from quasars using FIRST survey data has already been reported (Wadadekar \& Kembhavi 1999).

\section{Radio/optical comparisons}

We compare the positions of AGN listed in VV01 to the positions of radio sources in the FIRST radio source catalog (April 11, 2003 version publicly available at http://sundog.stsci .edu/), and calculate the angular separation between each AGN and each FIRST source. About $3.6 \%$ of sources in the FIRST catalog have been tagged as possible sidelobes of bright sources. Of these, $<10 \%$ are real sources and considerably less than $1 \%$ of the unflagged sources in the catalog are sidelobes (White et al. 1997). We have excluded all such flagged sidelobe sources from our cross correlation. We are then left with a total of 781667 unflagged sources in the northern and southern strips, covering a total area of about 9033 square degrees. On an average, there are 86.53 unflagged FIRST sources per square degree of sky.

In order to find coincidences between VV01 and FIRST sources, we adopt the straightforward methodology used by Wadadekar \& Kembhavi (1999). We begin with a search circle of radius of 600 arcsec centered on each VV01 AGN, and look for FIRST radio sources within this circle. This search radius is clearly too large, given the uncertainties in the radio and optical positions. We choose this large trial radius only to robustly estimate chance coincidences. When there is more than one FIRST source in the search circle, we tentatively count all such sources as matches. In Fig. 1 we show a histogram of the angular separation between the VV01 AGN and the FIRST sources found within the search circles.

To see which of the radio sources found can be accepted as true identifications, we estimate the AGN-FIRST source chance coincidence rate for a random distribution of FIRST survey sources using a technique identical to the one discussed in Wadadekar \& Kembhavi (1999). The straight line in Fig. 1 is the expected number of chance coincidences between AGN and FIRST sources, in annuli of radius shown on the abscissa and width 5 arcsec around the AGN. It is seen from the figure that the expected number of chance coincidences closely matches the number of actual coincidences for radius $\geq 60$ arcsec, indicating that most FIRST sources found more than 60 arcsec away from the AGN are chance coincidences. 


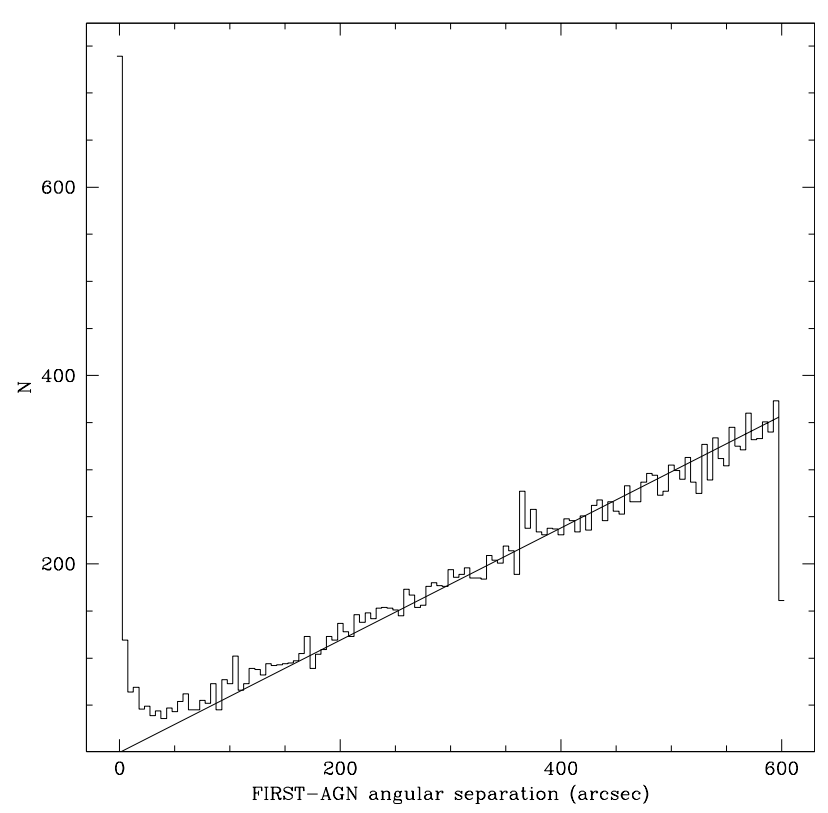

Fig. 1. A histogram of the angular separation between the AGN and the corresponding FIRST source. The straight line is the number of chance matches expected, in annuli of radius shown on the $X$-axis having a width of 5 arcsec, if the FIRST sources were randomly distributed in the sky.

On the other hand, matches within 3 arcsec are almost all real (less than $0.05 \%$ chance identification probability).

The high resolution and excellent astrometric accuracy of the FIRST survey makes it well suited for studies of compact sources. Nevertheless, for our choice of cosmological parameters $\left(H_{0}=50 \mathrm{~km} \mathrm{~s}^{-1} \mathrm{Mpc}^{-1}\right.$ and $\left.q_{0}=0.5\right)$, for an overwhelming majority of AGN in our sample $(z<0.3)$, an angular size of 3 arcsec corresponds to a spatial scale of $<17 \mathrm{kpc}$, comparable to the typical radius of a spiral Galaxy. The astrometric error for FIRST sources depends on their flux. Point sources at the detection limit of the survey have positions accurate to better than 1 arcsec at $90 \%$ confidence; $2 \mathrm{mJy}$ point sources in typically noisy regions have positions good to 0.5 arcsec. VV01 positions are compiled from the wide astronomical literature on AGN and mean positional errors are impossible to estimate. However, most of the brighter galaxies listed have positions accurate to within an arcsec. Given the combined root mean square error in FIRST and VV01 positions, it is possible to claim that the radio emission discussed here emanates from the galaxy concerned but does not allow us to localise the emission within the Galaxy. Consequently, it is not possible to distinguish nuclear emission from emission emanating from star-forming regions situated away from the center of the galaxy. We use a search circle of radius 3 arcsec and count all matches found within this radius as true matches. All subsequent discussion about the radio properties of the AGN only uses matches obtained with this search radius.

We have 775 AGN with a FIRST source located less than 3 arcsec away from the centre of the AGN. Only 1 AGN (NGC 1068) has two FIRST sources within three arcsec of its centre. Note that these "AGN" include all objects listed in Table 3 of VV01. Some of our detections are actually
Table 2. Number of radio detections of various classes of AGN at the 1 mJy flux limit of the FIRST survey.

\begin{tabular}{lll}
\hline \hline Class & \multicolumn{2}{l}{$\begin{array}{l}\text { Number in area covered Number detected } \\
\text { by FIRST }\end{array}$} \\
\hline Seyfert 1 & 788 & 188 \\
Intermediate type Seyferts & 434 & 122 \\
Seyfert 2 & 298 & 179 \\
Seyfert 3 (LINERS) & 63 & 41 \\
Unconfirmed Seyferts $^{a}$ & 190 & 125 \\
H-II regions $_{\text {Unlisted }^{b}}$ & 80 & 39 \\
\hline
\end{tabular}

a Many of these tentative identifications are from White et al. (2000) and will require further spectroscopic observations to confirm their status.

${ }^{b}$ These sources do not have a class listed in the VV01 catalog.

$\mathrm{H}-\mathrm{II}$ regions, galaxies with unclassified spectra and LINERS (some of which may be starburst powered). There are 2068 non detections, amongst the VV01 AGN covered by FIRST, and we assign an upper limit of $1 \mathrm{mJy}$ to their radio flux at $1.4 \mathrm{GHz}$. Table 1 provides a summary of our radio detections. Table 2 lists the number of detections for the various types of AGN listed in VV01.

The radio and optical properties of the AGN with detections are summarized in Table 3 . The columns are follows:

- Column 1: the most common name for the object as listed in VV01. The 214 radio detections with a $*$ preceding their designation have not been previously detected as a radio source (according to VV01) and this work most likely represents the first radio detection of the objects. The lack of previous radio detection is either because the object had not been observed or because its flux was lower than the sensitivity limit reached by previous radio observations of that object. There is therefore no uniform upper limit to the flux density of these previously undetected objects. Nevertheless, a overwhelming majority $(93 \%)$ of the new detections have a integrated radio flux density $<20 \mathrm{mJy}$;

- Column 2: object class as listed in VV01;

- Column 3: the redshift from VV01, obtained from the literature. $\mathrm{A} *$ in front of the redshift means that it has been estimated from a low dispersion slitless spectrum and is of lesser accuracy;

- Column 4: the photoelectric or photographic $V$ magnitude. Where $V$ magnitudes are not available other magnitudes are listed. These include Photographic $\left(^{*}\right)$, red $(R)$, photographic $\mathrm{O}$ - or J- plates $(\mathrm{O})$;

- Columns 5 and 6: coordinates for the object from VV01;

- Columns 7 and 8: peak and integrated radio flux densities for the radio source from the FIRST catalog in mJy;

- Column 9: angular separation between AGN and FIRST source in arcsecond.

The optical properties of the non-detections (upper limits) are summarized in Table 4. The columns are identical to those in Table 3 except that the last three columns (which contain radio data) are missing. 


\section{Radio-optical luminosity corellations}

We have shown in Fig. 2 a plot of the $5 \mathrm{GHz}$ radio luminosity against the logarithm of the redshift for the AGN. Throughout this paper, we compute luminosities and absolute magnitudes using optical and radio spectral indices, $\alpha_{\mathrm{op}}=\alpha_{\mathrm{r}}=-0.5$ with $L(v) \propto v^{+\alpha}$. The non-detections are shown as dots, and form the almost continuous lower envelope which indicates the radio luminosity corresponding to a radio flux of $1 \mathrm{mJy}$ over the redshift range shown. The triangles indicate Seyfert 1 (S1, S1h, S1i, S1n), plus signs indicate intermediate type Seyfert galaxies (S1.0, S1.2, S1.5, S1.8, S1.9; see the introductory material accompanying VV01 for the exact definition of each AGN category), the open circles indicate Seyfert 2 galaxies (S2), the asterisks indicate Seyfert 3 (LINER) galaxies (S3, S3b, S3h), crosses indicate $\mathrm{H}-\mathrm{II}$ regions (H2 and HP), while the filled squares indicate those sources for which the category is either not listed or deemed suspect (S, S?, ?, blank) in VV01. The same symbols are used in all subsequent figures of this paper.

Seyfert 1 and Seyfert 2 galaxies span the entire redshift range shown in the figure. However, $\mathrm{H}-\mathrm{II}$ regions and LINERS are predominantly seen at low redshifts. This is because the low average radio luminosity of these objects makes them detectable above the FIRST flux limit only at low redshifts. Another trend seen is that many of the highest redshift AGN do not have a classification in VV01 (these are indicated by filled squares). Many of these objects are in reality moderate redshift quasars, which have been included in Table 3 of VV01, only because they have absolute magnitude $M_{B}>-23$.

In Fig. 4 is shown a plot of the $5 \mathrm{GHz}$ radio luminosity against the absolute magnitude for the radio detections. For our set of 775 AGN with radio detections, 688 have a $V$ magnitude listed in VV01. (NGC 1266 has a incorrect magnitude of 0.0, 14 objects have no magnitude listed and 72 objects some other magnitude listed). We only plot the points with a $V$ magnitude available in this figure. There is a clear correlation between the logarithm of the radio luminosity and absolute magnitude. The formal linear correlation coefficient for the Seyfert 1 is -0.46 while that for the Seyfert 2 is -0.56 . Both correlations are significant at the $>99.9 \%$ confidence level.

However, it is seen from Figs. 3 and 2 that mean radio and optical luminosity both increase with redshift, which is due to the existence of a limiting radio flux and apparent magnitude in the surveys in which quasars are discovered. It is possible that the observed correlation between radio and optical luminosities is mainly due to the separate dependence of each luminosity on the redshift $z$. It is important to see if the correlation remains significant when such an effect of the redshift on the observed correlation is taken into account. This is done by evaluating a partial linear correlation coefficient as discussed in Wadadekar \& Kembhavi (1999).

For the Seyfert 1, the partial linear correlation coefficient is -0.13 , which is significant at only at the $97.6 \%$ confidence level. This is at variance with the findings of Ho \& Peng (2001) who reported a significant correlation between nuclear radio and optical emission for their sample of Seyfert 1. This discrepancy can be explained by the very large range in absolute magnitude spanned by the nuclear component of galaxies in their

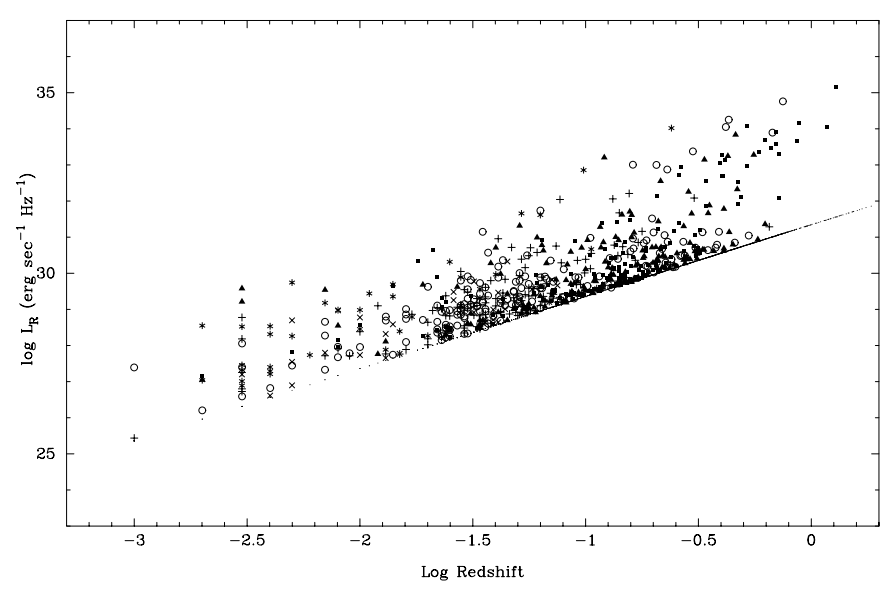

Fig. 2. Radio luminosity as a function of redshift. The various symbols used correspond to the various kinds of AGN, as explained in the text. The lower locus of dots indicates the $1 \mathrm{mJy}$ upper limits.

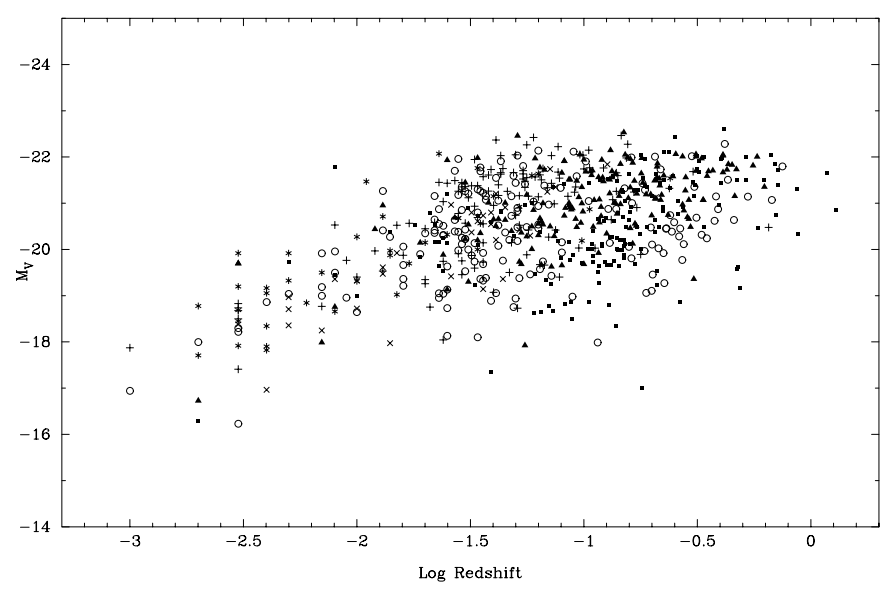

Fig. 3. Absolute magnitude of the AGN in our sample as a function of redshift. The symbols are as in Fig. 2.

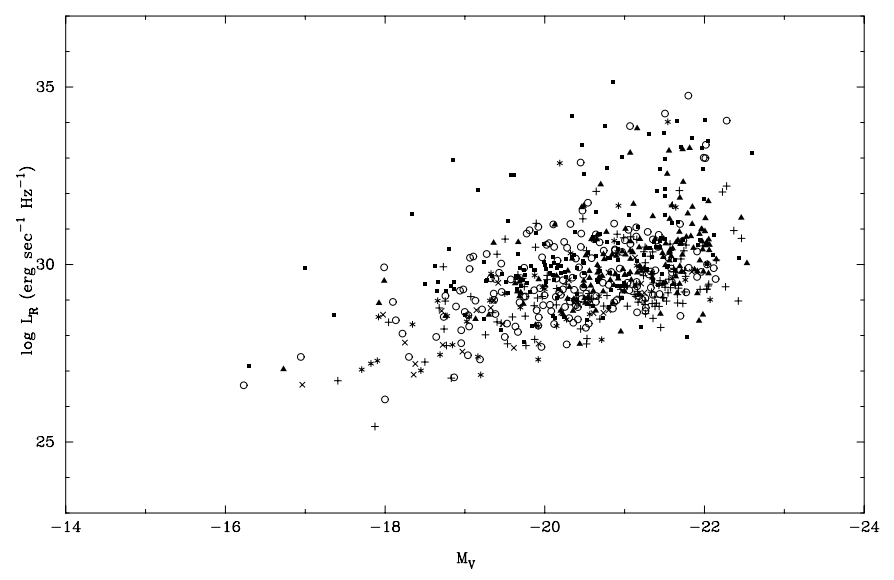

Fig. 4. Radio luminosity as a function of absolute magnitude. The symbols are as in Fig. 2.

sample. The total (nuclear + bulge + disk) magnitude spans a much narrower range in absolute magnitude and any correlation with radio emission is consequently much weaker.

For the Seyfert 2, the partial correlation coefficient is 0.13 which is significant at the $98.7 \%$ level. 
Table 5. Radio luminosity, size and spectral index for Seyfert 1 and Seyfert 2 galaxies.

\begin{tabular}{lcc}
\hline \hline & Seyfert 1 & Seyfert 2 \\
\hline Mean log radio luminosity-detections only $\left(\mathrm{erg} \mathrm{s}^{-1} \mathrm{~Hz}^{-1}\right)$ & $30.24 \pm 1.14$ & $29.73 \pm 1.41$ \\
Mean log radio luminosity-detections and upper limits $\left(\mathrm{erg} \mathrm{s}^{-1} \mathrm{~Hz}^{-1}\right)$ & $28.71 \pm 0.11$ & $28.99 \pm 0.11$ \\
Mean radio source size $(\mathrm{kpc})$ & $11.93 \pm 1.95$ & $5.79 \pm 0.97$ \\
Mean radio spectral index $\left(\alpha_{6}^{20}\right)$ & $-0.02 \pm 1.00$ & $0.15 \pm 1.44$ \\
\hline
\end{tabular}

The lack of a highly significant radio-optical luminosity correlation for both classes of Seyferts is not altogether surprising, because the radio emission is very likely linked to the presence of an active nucleus (or starburst) while the optical emission has significant contribution from stars in the bulge and disk of the underlying Galaxy. In fact, recent work indicates that the nuclear component may contribute as little as $0.01 \%$ of the optical continuum emission of Seyfert galaxies (Ho \& Peng 2001). On the other hand, a significant fraction of the radio emission from AGN likely originates in the nuclear region.

\section{Radio luminosity, source size and spectral index}

\subsection{Radio luminosity}

We have FIRST detections for 188 out of the 788 Seyfert 1 and 179 out of the 298 Seyfert 2 galaxies in our sample. We have used the Seyfert classifications listed in the VV01 catalog, without attempting to verify them independently. The radio detection rate is thus about $25 \%$ for the Seyfert 1 and $60 \%$ for the Seyfert 2 at the 1 mJy level.

We list in Table 5 the mean radio luminosity for the two classes of Seyferts. Errors listed are the root mean square deviations in the radio luminosity. The mean radio luminosity of both types of Seyferts is two orders of magnitude lower than even the optically selected quasars, most of which fall in the category of radio quiet quasars. Though the radio emission from Seyferts is weak, it is still two orders of magnitude higher than the typical emission from normal galaxies (Wunderlich et al. 1987). The table indicates that the mean radio luminosity of the Seyfert 1 is greater than that of the Seyfert 2, but this difference in mean radio luminosity is likely caused by a bias in redshift - the Seyfert 2 have a lower mean redshift $\left(z_{\text {mean }}=0.11\right)$ compared to the Seyfert 1 galaxies $\left(z_{\text {mean }}=0.18\right)$. Such a distance-bias has been noted previously (Dahari \& de Robertis 1988; Roy et al. 1994). It arises in all optically selected Seyfert samples since Seyfert 2's are less luminous optically than Seyfert 1. However the standard deviation of the radio luminosity is higher than the difference in mean luminosities and therefore a strong statistical conclusion cannot be drawn from our data. A Gehan-Wilconox test reveals that there is a $1 \%$ probability that the Seyfert 1 and Seyfert 2 are drawn from the same parent population. This seems to indicate that the two Seyfert populations are drawn from different parent populations or at least have a strong selection bias.

It should be noted that a significant fraction of Seyfert galaxies in the FIRST area only have an upper limit on their radio flux and radio luminosity. When we wish to estimate the mean for a quantity that includes both known values and upper limits, the Kaplan-Meier estimator may be used. This estimator requires that the censoring be random. Two factors contribute to making the censoring in radio luminosity random: 1. our radio observations are of a sample of Seyfert galaxies selected mostly using observations at non-radio wavelength and the radio and optical luminosities are not corelated and 2. the galaxies we are considering lie at different distances contributing to an increased randomness in radio luminosity based censoring.

We list in Table 5 the mean-radio luminosity of (detections + non-detections) for the Seyfert 1 and Seyfert 2 galaxies. When non-detections are accounted for, the K-M estimator predicts a slightly higher radio luminosity for the Seyfert 1 relative to the Seyfert 2. The error listed is that in the estimate of the mean, obtained using the Kaplan-Meier estimator.

Ulvestad \& Ho (2001) obtain similar results both with regard to Seyfert 1's being more luminous than Seyfert 2's (although their sample is not distance biased) and the results of the Gehan-Wilcoxon test. They suggest that this difference in radio luminosities can be accommodated in the unified scheme provided a minimum level of Seyfert activity is required for the radio source to emerge from the vicinity of the active nucleus. For our sample, the redshift bias (Seyfert 1 have higher mean redshift) is sufficient to explain the small difference in mean radio luminosities.

\subsubsection{Normalized radio luminosities}

Edelson (1987) found no significant difference between the radio luminosities of Seyfert 1 and Seyfert 2 galaxies in their sample. Nevertheless, when radio luminosities were normalized by the total optical luminosity, Seyfert 2 galaxies were found to be, on the average, twice as luminous as Seyfert 1 galaxies, at the $99 \%$ confidence level. We used the radio to optical luminosity ratio $R$ as a measure of normalized radio luminosities for the the two types of Seyferts. For Seyfert 1 galaxies the mean value of $\log R$ is $0.95 \pm 1.00$, while for Seyfert 2 galaxies, the mean value of $\log R$ is $0.73 \pm 1.19$. In fact, we find that the mean normalized radio luminosity of the Seyfert 1 is somewhat larger than that of the Seyfert 2. However, the small difference in mean normalized radio luminosity is almost certainly due to the optical obscuration of Seyfert 2 nuclei and is not intrinsic.

\subsection{Radio source sizes}

An expectation from the unified scheme is that radio source sizes of Seyfert 1's should be systematically smaller than those of Seyfert 2's due to foreshortening effects. Of course, the measured source size will in general depend on the total radio flux 
of the source; this effect can be roughly accounted for by using the FWHM of the radio source as a measure of the size of the radio source. We used the deconvolved major axis of the FIRST radio source as a measure of radio source size.

Another complication is that a significant number of our radio detections of Seyferts $(16 \%)$ are unresolved in the FIRST survey and are listed with a deconvolved major axis source size of 0.00 arcsec in the FIRST catalog. 45 out of 188 Seyfert 1 and 22 out of 179 Seyfert 2 galaxies show unresolved radio emission. Additionally, the uncertainty in radio source size depends upon the total flux of the source. Also, the beam size varies as a function of the position of the source in the sky, so the upper limits on the sizes of unresolved sources are not uniform. In such a situation, it is nearly impossible to measure radio source sizes reliably. Nevertheless, we use the K-M estimator to compare the source sizes of Seyfert 1 and Seyfert 2 galaxies. We used the FIRST beam size of 5.4 arcsec as the upper limit for sources that were unresolved. The mean of the radio source sizes was calculated using the Kaplan-Meier estimator, which gave $11.931 \pm 1.949 \mathrm{kpc}$ and $5.794+/-0.572 \mathrm{kpc}$ for Seyfert 1's and Seyfert 2's respectively. The error listed is the error in the estimate of the mean, obtained using the Kaplan-Meier estimator. Prima facie, this seem to run counter to the predictions of the unified scheme. But the same subtle biases in redshift and luminosity may also operate here. Perhaps more importantly, the angular resolution of the FIRST data is insufficient to measure sizes of small scale radio jets of the kind that operate in radio-weak AGN, except in the nearest Seyferts. Also, the resolved emission in the FIRST survey in some cases, may be caused by galaxy-scale star formation, rather than AGN emission.

\subsection{Radio spectral index}

Radio flux measurements at $6 \mathrm{~cm}$, as listed in VV01 were used to compute radio spectral index for the Seyfert galaxies. Note that only a few galaxies have $6 \mathrm{~cm}$ fluxes available. Also in general, the $20 \mathrm{~cm}$ (FIRST) and $6 \mathrm{~cm}$ measurements are not made with "matched beams". This means that data at the two wavelengths may well be probing emission over different parts of the Galaxy. Any conclusions that one may draw from measurements of spectral indices are therefore subject to considerable uncertainty.

The mean spectral index of Seyfert 1 is $-0.02 \pm 1.00$ (assuming $L_{v} \propto v^{+\alpha}$ ) and that of Seyfert 2 is $0.15 \pm 1.44$. The error estimates are the root mean square deviation of the spectral index. There is a slight indication that the Seyfert 2 have larger radio spectral indices than the Seyfert 1. But the difference between the spectral indices of the two populations as measured by a Gehan-Wilcoxon gives a 77\% probability that the two populations are the same.

\section{Conclusions}

The main results of this paper are:

- We have detected nuclear radio emissions from 775 AGN of which 214 were previously undetected in the radio. $\sim 27 \%$ of the known AGN within the 9033 square degrees of sky covered by FIRST have a flux higher than $1 \mathrm{mJy}$.

- We do not find a statistically significant correlation between radio luminosity of Seyfert 1 and Seyfert 2 and the optical luminosity of the underlying host Galaxy. The apparent correlation seen is induced largely by the effects of redshift.

- We find that mean radio luminosities of detected Seyfert 1 and Seyfert 2 are consistent with expectations from the unified scheme. When we compute the Kaplan Meier estimator for detections and non-detections for the two Seyfert types, they show a small difference in radio luminosity distribution. When normalised by optical luminosity, the radio luminosities of the two Seyfert populations are not significantly different. We also find that radio source sizes in Seyfert 1 are significantly larger than Seyfert 2, but this result is subject to numerous caveats. The limited radio spectral index measurements indicate that Seyfert 1 and Seyfert 2's are drawn from the same parent population.

Acknowledgements. The author wishes to thank Ajit Kembhavi for useful discussions and the referee James Ulvestad for insighful comments that helped improve this paper. The author's research at the IAP was supported by the Indo-French centre for promotion of advanced scientific research (CEFIPRA) through grants 1610-1 and 1910-1.

\section{References}

Becker, R. H., White, R. L., \& Helfand, D. J. 1995, ApJ, 450, 559 de Bruyn, A. G., \& Wilson, A. S. 1976, A\&A, 53, 93

de Bruyn, A. G., \& Wilson, A. S. 1978, A\&A, 64, 433

Condon, J. J., Cotton, W. D., Greisen, E. W., et al. 1998, AJ, 115, 1693

Dahari, O., \& de Robertis, M. M. 1988, ApJS, 67, 249

Edelson, R. A. 1987, ApJ, 313, 651

Ho, L. C., \& Peng, C. Y. 2001, ApJ, 555, 650

Roy, A. L., Norris, R. P., Kesteven, M. J., Troup, E. R., \& Reynolds, J. E. 1994, ApJ, 432, 496

Schmitt, H. R., Antonucci, R. R. J., Ulvestad, J. S., et al. 2001, ApJ, 555,663

Ulvestad, J. S., \& Wilson, A. S. 1984, ApJ, 285, 439

Ulvestad, J. S., \& Wilson, A. S. 1989, ApJ, 343, 659

Ulvestad, J. S., \& Ho, L. C. 2001, ApJ, 558, 561

Veron-Cetty, M. P., \& Veron, P. 2001, A\&A, 374, 92

Wadadekar, Y., \& Kembhavi, A. 1999, AJ, 118, 1435

White, R. L., Becker, R. H., Helfand, D. J., \& Gregg, M. D. 1997, ApJ, 475,479

White, R. L., Becker, R. H., Gregg, M. D., et al. 2000, ApJS, 126, 133

Wunderlich, E., Wielebinski, R., \& Klein, U. 1987, A\&AS, 69, 487 